However, there are challenges in setting up and undertaking research in a non-NHS setting which can present perceived barriers to participation.

Aim To identify the challenges and perceived barriers and to support organisations, including non-NHS, who wish to participate in research by providing them with knowledge, skills and tools to de-mystify and simplify the process, thus supporting them in their research participation.

Methods

- Working with the Local Clinical Research Network to identify the challenges and potential barriers to setting up and undertaking research in organisations with little or no research experience, which includes some non-NHS organisations. This involved communications with all hospices within the East Midlands and asking them what they wanted and what would be useful to them;

- Development of a suite of research governance Host Organisation Standard Operating Procedures to include governance arrangements for the delivery of research in a non-NHS setting;

- Close collaboration with the local NHS Trust to ensure alignment of governance processes.

Conclusions Although there are challenges and perceived barriers to delivering research within a non-NHS setting, none of these are insurmountable with the correct level of support. It is important that these organisations are able to participate in research if they wish to, secure in the knowledge that they are well supported and have the appropriate arrangements in place to allow the effective and safe delivery of the research. It is also important that patients, families and healthcare professionals in palliative and end of life care organisations are offered the opportunity to participate in research if they would like to.

\section{P-109 RANDOMISED CLINICAL TRIALS IN A HOSPICE SETTING: TOIL, TRIBULATIONS, TRIUMPH}

${ }^{1,2}$ Charlie Hall, ${ }^{1} J a n e$ Cook, ${ }^{1}$ Honor Blackwood, ${ }^{1}$ Erna Haraldsdottir, ${ }^{1}$ Duncan Brown, ${ }^{3}$ Matthew Maddocks, ${ }^{4}$ Liz Dixon, ${ }^{5}$ Richard Skipworth, ${ }^{2}$ Marie Fallon, ${ }^{1,2}$ Barry Laird. ${ }^{1} S t$ Columba's Hospice, Edinburgh, UK; ${ }^{2}$ The University of Edinburgh, Edinburgh, UK; ${ }^{3} \mathrm{Cicely}$ Saunders Institute of Palliative Care, Policy and Rehabilitation, London, UK; ${ }^{4}$ Southampton Clinical Trials Unit, Southampton, UK; ${ }^{5}$ Royal Infirmary, Edinburgh, UK

\subsection{6/bmjspcare-2019-HUKNC.132}

Background Despite clinical research being advocated as a key component of palliative care (Payne, Preston, Turner \& Rolls, 2013), most patients under the care of palliative care services will not have the opportunity to participate in research. Of the 200 plus UK hospices, few patients get the opportunity to actively participate in research for multiple reasons including lack of expertise, funding, opportunity and anxiety about excess patient burden. However even patients with advanced disease describe multiple benefits from being involved in research trials (Middlemiss, Lloyd-Williams, Laird \& Fallon, 2015) and it is possible to establish and conduct clinical trials within the hospice setting.

Aim To describe the experience of establishing and conducting a randomised clinical trial in an independent UK hospice.

Results The ethical, logistical and regulatory hurdles to establishing a clinical trial are described and include local engagement, funding, sponsorship, developing academic links and tackling barriers to recruitment. Novel initiatives are described, including volunteer and carer engagement and motivating team members to realise the advantages of in-house clinical trials. Further, the image of hospice care was improved through the establishment of a research culture.

Conclusions It is possible to establish and conduct clinical trials within a UK hospice setting; and this has multiple advantages to patients and staff alike.

\section{P-110 CHILDREN'S PALLIATIVE CARE: AN EXPLORATION OF THE UNMET NEEDS OF CHILDREN AND THEIR FAMILIES}

Georgina Constantinou, Gurch Randhawa, Erica Cook. University of Bedfordshire, Luton, UK

\subsection{6/bmjspcare-2019-HUKNC.133}

Background Understanding the needs of children with life-limiting illnesses and their families has been highlighted as a top research priority. Children's palliative care services have been shown to have limited funding and resources to provide services which are equipped to meet the needs of families. There are, however, few studies which outline whether or not services, from the perspective of professionals and parents, meet the needs of this group.

Aim This study aims to examine how children's palliative services care can be improved to meet the needs of children and their families.

Methods Phenomenological research eliciting semi-structured interviews with 29 professionals delivering care to children with life-limiting conditions were conducted. Professionals were recruited from a variety of services in the children's palliative care pathway; hospice, hospitals, community care teams, social services, occupational therapy, spiritual services, supportive care, complementary therapy and so on. Interviews were recorded, transcribed and analysed thematically.

Proposed importance of the findings These findings provide insight into the professional experiences of unmet palliative care needs. Professional experiences present necessary improvements across the sampled palliative care services. Alongside interviews with parents currently accessing services and those bereaved parents, perspectives will be triangulated to highlight the unmet need. Recommendation for policy and guidance will be made to suggest improvements to the care of families in the future.

\section{P-111 MULTI-DISCIPLINARY JOURNAL CLUB TO SUPPORT A WHOLE-HOSPICE RESEARCH CULTURE}

Tom Steele, Sarah Stanley, Amara Callistus Nwosu. Marie Curie Hospice, Liverpool, UK

\subsection{6/bmjspcare-2019-HUKNC.134}

Research in palliative care is essential to improve outcomes for patients with terminal illness. It is important that hospices are central to research in the speciality, however, research is not always well embedded in hospice cultures. Essential to this is raising awareness amongst team members of all disciplines, both of the importance of research in general and also the role of evidence in underpinning clinical practice.

Journal clubs are a well-established way of sharing research within teams and developing critical appraisal skills 